\title{
Effect of direct-fed microbials on culturable gut microbiotas in broiler chickens: a meta-analysis of controlled trials
}

\author{
Chhaiden Heak', Peerapol Sukon ${ }^{1,2, *}$, and Pairat Sornplang ${ }^{1}$
}

* Corresponding Author: Peerapol Sukon Tel: +66-43-202-404, Fax: +66-43-202-404,

E-mail: sukonp@kku.ac.th

${ }^{1}$ Faculty of Veterinary Medicine, Khon Kaen University, Khon Kaen 40002, Thailand

2 Research Group for Animal Health Technology, Khon Kaen University, Khon Kaen 40002, Thailand

ORCID

Chhaiden Heak

https://orcid.org/0000-0003-0111-255X

Peerapol Sukon

https://orcid.org/0000-0002-0899-2572

Pairat Sornplang

https://orcid.org/0000-0001-8053-7091

Submitted Dec 29, 2017; Revised Feb 1, 2018; Accepted May 21, 2018
Objective: This meta-analysis was conducted to evaluate the overall effect of direct-fed microbial (DFM) or probiotic supplementation on the log concentrations of culturable gut microbiota in broiler chickens.

Methods: Relevant studies were collected from PubMed, SCOPUS, Poultry Science Journal, and Google Scholar. The studies included controlled trials using DFM supplementation in broiler chickens and reporting log concentrations of the culturable gut microbiota. The overall effect of DFM supplementation was determined using standardized mean difference (SMD) with a random-effects model. Subgroups were analyzed to identify pre-specified characteristics possibly associated with the heterogeneity of the results. Risk of bias and publication bias were assessed.

Results: Eighteen taxa of the culturable gut microbiota were identified from 42 studies. The overall effect of DFM supplementation on the log concentrations of all 18 taxa did not differ significantly from the controls $(\mathrm{SMD}=-0.06,95 \%$ confidence interval $[-0.16,0.04], \mathrm{p}=0.228$, $\mathrm{I}^{2}=85 \%, \mathrm{n}=699$ comparisons), but the 18 taxa could be further classified into three categories by the direction of the effect size: taxa whose log concentrations did not differ significantly from the controls (category 1), taxa whose log concentrations increased significantly with DFM supplementation (category 2), and taxa whose log concentrations decreased significantly with DFM supplementation (category 3). Category 1 comprised nine taxa, including total bacterial counts. Category 2 comprised four taxa: Bacillus, Bifidobacterium, Clostridium butyricum, and Lactobacillus. Category 3 comprised five taxa: Clostridium perfringens, coliforms, Escherichia coli, Enterococcus, and Salmonella. Some characteristics identified by the subgroup analysis were associated with result heterogeneity. Most studies, however, were present with unclear risk of bias. Publication bias was also identified.

Conclusion: DFM supplementation increased the concentrations of some beneficial bacteria (e.g. Bifidobacterium and Lactobacillus) and decreased those of some detrimental bacteria (e.g. Clostridium perfringens and Salmonella) in the guts of broiler chickens.

Keywords: Direct-fed Microbials; Probiotics; Broiler Chicken; Gut Microbiota; Systematic Review; Meta-analysis

\section{INTRODUCTION}

Direct-fed microbials (DFMs), or probiotics, are live microorganisms (mostly bacteria or yeast) that may confer health benefits on the host. DFM supplementation has been extensively studied in broiler chickens to replace or reduce the use of antibiotics as growth promoters. The mechanisms of DFMs for health benefits on the host are not fully understood and may involve several beneficial mechanisms [1]. Modification of the gut microbiota may be the most important mechanism, because the gut microbiota, a very complex microbial community, plays an important role in maintaining homeostasis and the physiology of the host, increasing health and productivity [2]. In addition to its direct contribution to host health, 
the gut microbiota of broiler chickens may also have indirect negative impacts on human and environmental health, because some microbes (as a part of gut microbiotas), such as Campylobacter spp. commonly shed and excreted in the feces, are harmful human pathogens [3]. These microbes may contaminate the food chain or environment and threaten human health.

Modification of the gut microbiota by increasing beneficial bacteria and decreasing harmful pathogens in the gastrointestinal tract is therefore the primary goal of DFM application in broiler chickens. DFM supplementation in broiler chickens can substantially alter (either increase or decrease) the populations of particular bacterial species in the gastrointestinal tract [4]. Recent advances in molecular techniques, especially next-generation sequencing, have led to a better understanding of the very diverse and complex communities of gut microbiotas in broiler chickens $[5,6]$, but most of the currently available evidence for the effect of DFMs on gut microbiotas have come from studies using culture-based techniques for determining concentrations of the gut bacteria of interest. We therefore conducted this systematic review and meta-analysis to determine the overall effect of DFM supplementation on the log concentrations of culturable gut microbiotas in broiler chickens and to identify pre-defined characteristics (factors) that may be associated with the heterogeneity of the results.

\section{MATERIALS AND METHODS}

\section{Review protocol}

The protocol for this study was developed using SYRCLE's protocol format [7] to minimize bias. This protocol is available in the supplementary information. Deviations from the protocol are identified in the relevant sections.

\section{Information sources and search strategy}

We collected relevant citations from PubMed, Scopus, Poultry Science Journal, and Google Scholar databases using the keywords: DFM, probiotic, microbiota, normal flora, and chicken. An example of a search algorithm for PubMed was: ((("probiotics"[MeSH Terms] OR "probiotics"[All Fields] OR "probiotic"[All Fields]) OR (direct[All Fields] AND fed[All Fields] AND microbial[All Fields])) OR ("microbiota"[MeSH Terms] OR "microbiota"[All Fields])) OR (normal[All Fields] AND ("Flora"[Journal] OR "flora"[All Fields]))) AND ("chickens" [MeSH Terms] OR "chickens"[All Fields] OR "chicken"[All Fields]). This study was limited to English publications. The last search was completed on 23 May 2016.

\section{Eligibility criteria and study selection}

Two independent reviewers assessed study eligibility with two screening steps. The reviewers first assessed the titles and abstracts of retrieved citations. Full-text articles were then assessed if the titles and abstracts passed the first screening step. The criteria for study selection were: i) randomized or non-randomized controlled trials with DFM supplementation as an intervention in broiler chickens, and ii) only studies reporting concentrations of the culturable gut microbiota either in the gastrointestinal tract or in the excreta. Studies were excluded with the following criteria: i) reviews, reports, errata, and duplicated articles, ii) not about broiler chickens, iii) trials with no control group and in vitro or in ovo studies, iv) treatments using non-viable DFMs, treatments other than DFMs (e.g. prebiotics, synbiotics, medicinal plants, or enzymes), or a combination of DFMs with other products, v) incomplete reporting of outcome data, and vi) trials with disease challenges or antimicrobial products. A PRISMA flow chart for systematic reviews was used to summarize the study selection.

\section{Study characteristics and data extraction}

Two independent reviewers extracted the available data from the included studies. The following data were collected for each study: i) bibliographic data (author names, publication year), ii) study design (number of animals, number of treatments and replicates, number of birds per pen or block), iii) characteristics of experimental chickens (broiler breed, sex, group sample size), iv) characteristics of intervention (DFM species and strains, product classification, administration dose, application frequency and route, duration of treatment), v) outcome measures (concentrations of culturable microbiota in the crop, small and large intestines, cecum, and excreta). Selection of the pre-defined characteristics or factors was based on information from the previous studies $[8,9]$. Some characteristics were used for subgroup analysis to explore heterogeneity of the results. Any inconsistencies between the reviewers were resolved by discussion.

\section{Assessment of risk of bias}

Two independent reviewers assessed the risk of bias for the included studies using SYRCLE's RoB tool [10]. The reported details of each study were evaluated for 10 categorical domains of bias: Selection bias (domains 1-3), Performance bias (domains 4 and 5), Detection bias (domains 6 and 7), Attrition bias (domain 8), Reporting bias (domain 9), and Others (domain 10) [10]. Each domain was assigned to one of three categories: "yes" indicated a low risk of bias, "no" indicated a high risk of bias, and "?" indicated an unclear risk of bias.

\section{Outcome measure and statistical analysis}

The outcome measure was the standardized mean difference (SMD) of the log concentrations of the culturable gut microbiota isolated from the chicken gastrointestinal tract (crop, small and large intestines, and cecum) or the excreta. Comprehensive Meta-Analysis version 3 (Biostat, Englewood, NJ, USA) was used for all analyses. Universal Desktop Ruler ver- 
sion 3 was used to convert graphically reported outcomes. Standard errors or standard errors of the mean were converted to standard deviations. As a pre-specified model of choice, a random-effects model was used for all analyses to obtain an overall or individual effect size with its 95\% confidence interval (CI). Differences were considered significant at $\mathrm{p}<0.05$. The heterogeneity of the results from the studies was assessed using Cochran's Q and qualified by $\mathrm{I}^{2}$. The percentage of $\mathrm{I}^{2}$ $<25 \%, 50 \%$ to $75 \%$, and $>75 \%$ indicated low, moderate, and high heterogeneity, respectively [11]. We identified 18 bacterial taxa of the culturable gut microbiota available for metaanalysis, so we classified these taxa into three categories based on the direction of the effect size to simplify the interpretation: taxa whose log concentrations did not differ significantly from the controls (category 1), taxa whose log concentrations increased significantly with DFM supplementation (category 2), and taxa whose log concentrations decreased significantly with DFM supplementation (category 3). This classification was not pre-specified in the study protocol.

Subgroup analysis was pre-specified for eight characteristics: broiler breed, sex, DFM product, DFM species, sampling organ, application frequency, application route, and application duration. Each characteristic was categorized into various subgroups (based on available information from the studies): broiler breed with eight subgroups (Arbor Acres, Cobb, Cuban EB24, Lingnan Yellow, Ross, Sasso X44, Tsukuba jidori, and unknown), sex with three subgroups (male, both male and female, and unknown), DFM product with two subgroups (commercial and non-commercial), DFM species with three subgroups (single, multiple, and unknown), sampling organ with seven subgroups (crop, duodenum, ileum, cecum, colon, excreta, and unknown), application route with four subgroups (feed, gavage, water, and both feed and water), application frequency with two subgroups (daily and once), and application duration with five subgroups $(\leq 7,>7$ and $\leq 14,>14$ and $\leq 21,>21$ and $\leq 14$, and $>42$ days of age). The subgroups were analyzed only if each subgroup contained at least four studies (or four outcome comparisons) [12] with at least two subgroups for each characteristic. Subgroups were analyzed only for Lactobacillus (chosen based on the large number of outcome comparisons and as a representative of beneficial bacteria) and for a combination of coliforms and Escherichia coli (E. coli) (chosen based on the large number of outcome comparisons and as representatives of detrimental bacteria) to reduce the number of subgroup comparisons and to simplify subgroup interpretation. This restriction was not pre-specified in the protocol but was based on available information after the primary meta-analysis. A sensitivity analysis was conducted to assess the robustness of the results because of decisions made during the systematic review; we evaluated the influences of model selection (random versus fixed). Publication bias was assessed visually using a funnel plot and was tested formally by Egger's test, with $\mathrm{p}<0.10$ indicating the presence of publication bias [13]. When publication bias was found, Duval and Tweedie's trim and fill methods were used to estimate the effect size of possibly missing publications [14].

\section{RESULTS}

\section{Search results and study selection}

A total of 1930 citations published between 1950 and 2016 were identified. Of these, 687 were identified as duplicates, and 1,094 did not pass the first screening step (Figure 1), so 149 full-text articles were assessed for study eligibility. Of these, 42 full-text articles (with a total of 699 outcome comparisons) were included for data extraction and meta-analysis. The references of all included studies are presented in the supplementary information (Supplementary Table S5).

\section{Characteristics of included studies}

The detailed characteristics of the 42 studies are provided in Supplementary Table S1 in the supplementary information. The 42 studies with 699 outcome comparisons included 18 culturable bacterial microbiota taxa (Table 1) and were published between 1996 and 2016. Most of the studies (36/42) were defined as randomized controlled trials. Ross was a broiler breed frequently used in the trials (22/42), and two studies reported unknown or unspecified breeds. Male chicks were frequently used in the trials (24/42), and an unknown or unspecified sex was occasionally found (11/42). Commercial DFM products were used in 17 studies. A single DFM species was used in 28 studies. The concentration of microbials used in treatments ranged from $10^{5}$ to $10^{12} \mathrm{cfu} / \mathrm{kg}$, and the microbials were normally mixed in feed (38/42) with daily supplementation (41/42).

\section{Primary analysis}

The effect of DFM supplementation on the log concentrations of the 18 bacterial taxa (based on the culture media) are also presented in Table 1 . The overall effect of DFM supplementation did not differ significantly between the 18 taxa and the controls (SMD $=-0.06,95 \%$ CI $[-0.16,0.04], \mathrm{p}=0.228, \mathrm{n}=$ 699 comparisons) and was highly heterogeneous among the studies $\left(\mathrm{Q}=4585.78, \mathrm{p}<0.001, \mathrm{I}^{2}=85 \%\right)$. Category 1 of the 18 taxa comprised nine taxa (aerobes, anaerobes, Bacteroides, Clostridium coccoides, Enterobacteriaceae, Gram-positive cocci, mesophilic bacteria, Streptococcus, and total bacterial counts); the overall effect of DFM supplementation did not differ significantly from the controls (SMD $=-0.12,95 \%$ CI $[-0.35$, $0.11], \mathrm{p}=0.298, \mathrm{n}=130$ comparisons) and were highly heterogeneous $\left(\mathrm{Q}=592.62, \mathrm{p}<0.001, \mathrm{I}^{2}=78 \%\right)$. Category 2 comprised four taxa (Bacillus, Bifidobacterium, Clostridium butyricum [C. butyricum], and Lactobacillus); the overall effect of DFM supplementation were associated with a significant increase 


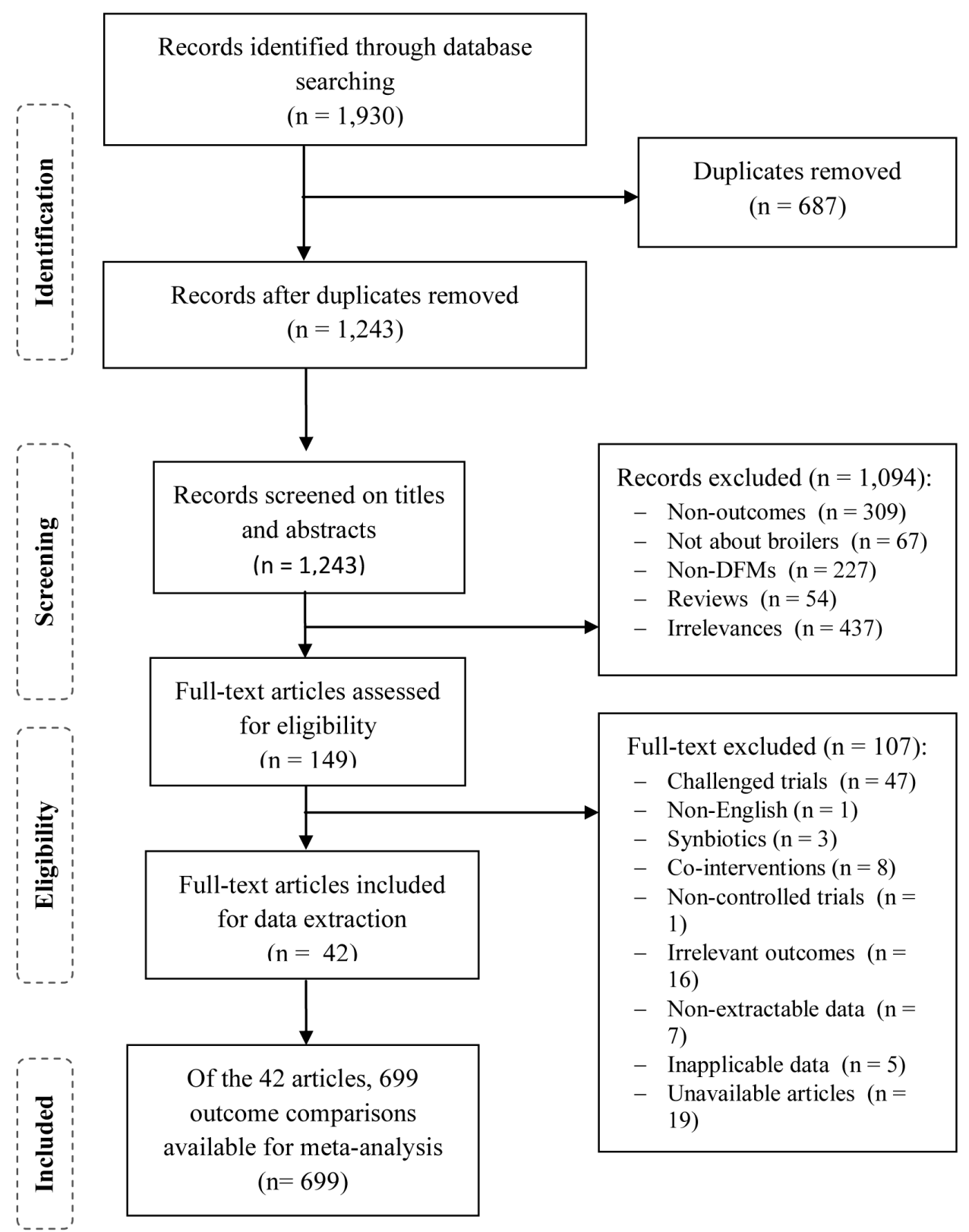

Figure 1. Flow diagram of study selection.

in the log concentrations of bacterial counts compared with the controls (SMD $=1.07,95 \% \mathrm{CI}[0.92,1.22], \mathrm{p}<0.001, \mathrm{n}=279$ comparisons) and were highly heterogeneous $(\mathrm{Q}=1,283.64$, $\mathrm{p}<0.001, \mathrm{I}^{2}=78 \%$ ). Category 3 comprised five taxa (Clostridium perfringens [C. perfringens], coliforms, E. coli, Enterococcus, and Salmonella); the overall effect of DFM supplementation was associated with a significant decrease in the log concentrations of bacterial counts compared with the controls (SMD $=-1.26,95 \% \mathrm{CI}[-1.42,-1.10], \mathrm{p}<0.001, \mathrm{n}=290$ comparisons) and were highly heterogeneous $\left(\mathrm{Q}=1,526.73, \mathrm{p}<0.001, \mathrm{I}^{2}=\right.$ $81 \%)$. As an example, a forest plot for the effects of DFM supplementation on the log concentrations of Salmonella is shown in Figure 2. Forest plots for the remaining bacterial taxa are provided in the supplementary information (Supplementary Figures S1-S5).

\section{Subgroup analysis}

The results of the subgroup analysis of eight characteristics are presented in Table 2 (for Lactobacillus) and 3 (for coliforms and E. coli). For Lactobacillus, three characteristics differed significantly among the subgroups: broiler breed, microbial species, and application duration ( $\mathrm{p}<0.001$ for each characteristic) (Table 2). For breed, the log concentration of Lactobacillus after DFM supplementation increased the most in Cuban EB24 ( $\mathrm{SMD}=2.13,95 \% \mathrm{CI}[0.85,3.42], \mathrm{p}<0.001, \mathrm{n}=6$ comparisons), followed by Ross, Arbor Acres, Cobb, and Lingnan Yellow. 
Table 1. Primary analysis of the effect of DFM supplementation on the log concentrations of gut microbiotas in broiler chickens

\begin{tabular}{|c|c|c|c|c|c|c|c|}
\hline \multirow{2}{*}{ Bacterial taxon } & \multirow{2}{*}{$n^{1)}$} & \multicolumn{3}{|c|}{ Effect size } & \multicolumn{3}{|c|}{ Heterogeneity } \\
\hline & & SMD & {$[95 \% \mathrm{Cl}]$} & $p$ value & Q & $p$ value & $\mathrm{I}^{2}$ \\
\hline \multicolumn{8}{|l|}{ Category $1^{2)}$} \\
\hline Aerobes & 19 & -0.02 & {$[-0.67,0.64]$} & 0.962 & 97.85 & $<0.001$ & 82 \\
\hline Anaerobes & 66 & -0.34 & {$[-0.70,0.02]$} & 0.066 & 367.94 & $<0.001$ & 82 \\
\hline Bacteroides & 4 & -0.35 & {$[-0.93,0.23]$} & 0.236 & 2.20 & 0.532 & 00 \\
\hline Clostridium coccoides & 2 & -0.03 & {$[-0.77,0.71]$} & 0.941 & 0.05 & 0.830 & 00 \\
\hline Enterobacteriaceae & 11 & 0.06 & {$[-0.53,0.65]$} & 0.839 & 30.23 & $<0.001$ & 67 \\
\hline Gram+ve cocci & 9 & 0.49 & {$[-0.29,1.27]$} & 0.217 & 31.40 & $<0.001$ & 75 \\
\hline Mesophilic bacteria & 8 & 0.64 & {$[-0.07,1.35]$} & 0.078 & 18.94 & 0.008 & 63 \\
\hline Streptococcus & 4 & -0.82 & {$[-1.66,0.01]$} & 0.054 & 4.76 & 0.190 & 37 \\
\hline Total bacterial counts & 7 & -0.10 & {$[-1.02,0.82]$} & 0.831 & 17.41 & 0.008 & 66 \\
\hline Overall & 130 & -0.12 & {$[-0.35,0.11]$} & 0.298 & 592.62 & $<0.001$ & 78 \\
\hline \multicolumn{8}{|l|}{ Category $2^{3)}$} \\
\hline Bacillus & 9 & 0.54 & {$[0.01,1.06]$} & 0.045 & 40.55 & $<0.001$ & 80 \\
\hline Bifidobacterium & 58 & 1.32 & {$[1.01,1.64]$} & $<0.001$ & 226.63 & $<0.001$ & 75 \\
\hline Clostridium butyricum & 7 & 5.11 & {$[2.84,7.39]$} & $<0.001$ & 47.64 & $<0.001$ & 87 \\
\hline Lactobacillus & 205 & 0.96 & {$[0.78,1.13]$} & $<0.001$ & 902.68 & $<0.001$ & 77 \\
\hline Overall & 279 & 1.07 & {$[0.92,1.22]$} & $<0.001$ & $1,283.64$ & $<0.001$ & 78 \\
\hline \multicolumn{8}{|l|}{ (ategory $3^{4)}$} \\
\hline C. perfringens & 58 & -1.60 & {$[-2.00,-1.21]$} & $<0.001$ & 341.14 & $<0.001$ & 83 \\
\hline Coliforms & 106 & -0.81 & {$[-1.05,-0.56]$} & $<0.001$ & 538.61 & $<0.001$ & 81 \\
\hline Escherichia coli & 83 & -1.20 & {$[-1.45,-0.94]$} & $<0.001$ & 304.50 & $<0.001$ & 73 \\
\hline Enterococcus & 8 & -1.21 & {$[-2.04,-0.38]$} & 0.004 & 33.00 & $<0.001$ & 79 \\
\hline Salmonella & 35 & -2.20 & {$[-2.70,-1.71]$} & $<0.001$ & 183.56 & $<0.001$ & 81 \\
\hline Overall & 290 & -1.26 & {$[-1.42,-1.10]$} & $<0.001$ & $1,526.73$ & $<0.001$ & 81 \\
\hline Overall effect of DFM & 699 & -0.06 & {$[-0.16,0.04]$} & 0.228 & $4,585.78$ & $<0.001$ & 85 \\
\hline
\end{tabular}

DFM, direct-fed microbial; SMD, standardized mean difference for the log concentrations; $\mathrm{Cl}$, confidence interval.

1) $n$, number of outcome comparisons.

2) Category 1, bacteria whose log concentrations did not differ significantly from the controls.

${ }^{3)}$ Category 2, bacteria whose log concentrations increased significantly with DFM supplementation.

4) Category 3, bacteria whose log concentrations decreased significantly with DFM supplementation.

For DFM species, the effect of DFM supplementation on the log concentrations of Lactobacillus was greatest for an unknown (unspecified) DFM species (SMD $=2.38,95 \%$ CI $[0.02,4.73]$, $\mathrm{p}=0.048, \mathrm{n}=5$ comparisons), followed by single and multiple DFM species. For application duration, the effect of DFM supplementation was greatest when applied between 21 and 42 days of age $(\mathrm{SMD}=1.23,95 \% \mathrm{CI}[0.98,1.49], \mathrm{p}<0.001, \mathrm{n}=$ 95 comparisons).

For the combination of coliforms and E. coli, three characteristics differed significantly among the subgroups: broiler breed, sampling organ, and application duration ( $\mathrm{p}<0.001$ for each characteristic) (Table 3). For broiler breed, the log concentrations of coliforms and E. coli decreased the most in an unknown (unspecified) breed ( $\mathrm{SMD}=-5.60,95 \% \mathrm{CI}[-7.87$, -3.33], $\mathrm{p}<0.001, \mathrm{n}=12$ comparisons), followed by Ross, Lingnan Yellow and Arbor Acres. For sampling organ, the log concentrations of coliforms and E. coli decreased the most in the colon (SMD $=-3.76,95 \%$ CI $[-5.26,-2.27], \mathrm{p}<0.001, \mathrm{n}=$ 14 comparisons), followed by the cecum and ileum. For application duration, the log concentrations of coliforms and E. coli decreased the most when the supplementation was applied between 21 and 42 days of age ( $\mathrm{n}=98$ comparisons, $\mathrm{SMD}=$ $-1.14,95 \%$ CI $[-1.39,-0.88], \mathrm{p}<0.001)$.

\section{Sensitivity analysis}

The results of the sensitivity analysis for comparing a randomeffects model (the model of choice for this study) with a fixedeffects model are presented in Table 4. The random-effects model found larger effect sizes than the fixed-effects model for all three categories of taxa, but the directions of the effect sizes and the results of the statistical tests were similar for all three categories. The SMDs (the magnitudes of the effect sizes) for the random-and fixed-effects models were -0.12 and -0.08 , respectively, for category 1 (taxa whose log concentrations did not differ significantly from the controls, $\mathrm{n}=130$ comparisons); 1.07 and 0.77 , respectively, for category 2 (taxa whose log concentrations increased significantly with DFM supplementation, $\mathrm{n}=279$ comparisons); and -1.26 and -0.89 , respectively, for category 3 (taxa whose log concentrations decreased significantly with DFM supplementation, $\mathrm{n}=290$ comparisons). 
Study name

Jeong and $\mathrm{KIm}, 2014-1$

Jeong and $\mathrm{Km}, 2014-2$

Jeong and Kim, 2014-3

Jeong and KIm, 2014-4

Jeong and Kim, 2014-5

Jeong and $\mathrm{KIm}, 2014-6$

Jeong and Kim, 2014-7

Jeong and $\mathrm{Km}, 2014-8$

Mohammadi Gheisar et al., 2016-1

Mohammadi Gheisar et al., 2016-2

Park and Km, 2014-1

Park and Kim, 2014-2

Park and Kim, 2014-3

Park and Kim, 2014-4

Park and Kim, 2014-5.

Park and Kim, 2014-6

Salim et al., 2013-1

Salim et al., 2013-2

Yang et all, 2012-1

Yang et all, 2012-2

Yang et al., 2012-3

Yang et all., 2012-4

Yang et al., 2012-5

Yang et all, 2012-6

Yang et all., 2012-7

Yang et al., 2012-8

Yang et all, 2012-9

Yang et al., 2012-10

Yang et al., 2012-11

Yang et al., 2012-12

Yang et al., 2012-13

Yang et al., 2012-14

Yang et al., 2012-15

Yang et al., 2012-16

Yang et al., 2012-17

\begin{tabular}{|c|c|c|c|c|}
\hline \multirow[b]{2}{*}{$\begin{array}{l}\text { Std diff } \\
\text { in means }\end{array}$} & \multicolumn{3}{|c|}{ Statistics for each study } & \multirow[b]{2}{*}{ p-Value } \\
\hline & $\begin{array}{l}\text { Lower } \\
\text { limit }\end{array}$ & $\begin{array}{c}\text { Upper } \\
\text { limit }\end{array}$ & Z-Value & \\
\hline-1.94 & -2.91 & -0.97 & -3.92 & 0.000 \\
\hline-3.01 & -4.18 & -1.84 & -5.05 & 0.000 \\
\hline-2.28 & -3.31 & -1.25 & -4.35 & 0.000 \\
\hline-2.81 & -3.94 & -1.68 & -4.88 & 0.000 \\
\hline-2.87 & -4.01 & -1.73 & -4.93 & 0.000 \\
\hline-3.83 & -5.17 & -2.48 & -5.57 & 0.000 \\
\hline-0.92 & -1.77 & -0.08 & -2.15 & 0.031 \\
\hline-2.08 & -3.07 & -1.09 & -4.10 & 0.000 \\
\hline-1.03 & -1.96 & -0.10 & -2.16 & 0.031 \\
\hline-1.19 & -2.14 & -0.24 & -2.45 & 0.014 \\
\hline-3.28 & -4.51 & -2.06 & -5.25 & 0.000 \\
\hline-3.88 & -5.23 & -2.52 & -5.60 & 0.000 \\
\hline-3.53 & -4.81 & -2.25 & -5.41 & 0.000 \\
\hline-3.06 & -4.23 & -1.88 & -5.08 & 0.000 \\
\hline-2.72 & -3.83 & -1.61 & -4.80 & 0.000 \\
\hline-2.89 & -4.03 & -1.75 & -4.95 & 0.000 \\
\hline-1.07 & -2.12 & -0.02 & -2.01 & 0.045 \\
\hline-1.40 & -2.49 & -0.31 & -2.51 & 0.012 \\
\hline-0.20 & -1.34 & 0.93 & -0.35 & 0.726 \\
\hline-0.36 & -1.50 & 0.78 & -0.62 & 0.537 \\
\hline-0.04 & -1.17 & 1.09 & -0.07 & 0.943 \\
\hline-6.25 & -8.99 & -3.50 & -4.46 & 0.000 \\
\hline-2.45 & -3.95 & -0.95 & -3.21 & 0.001 \\
\hline-4.32 & -6.39 & -2.26 & -4.10 & 0.000 \\
\hline-0.33 & -1.47 & 0.81 & -0.57 & 0.570 \\
\hline-0.46 & -1.60 & 0.69 & -0.78 & 0.435 \\
\hline-0.32 & -1.46 & 0.82 & -0.55 & 0.581 \\
\hline-9.38 & -13.30 & -5.46 & -4.69 & 0.000 \\
\hline-3.51 & -5.31 & -1.70 & -3.81 & 0.000 \\
\hline-7.25 & -10.37 & -4.14 & -4.56 & 0.000 \\
\hline-0.47 & -1.62 & 0.68 & -0.80 & 0.423 \\
\hline-0.56 & -1.71 & 0.59 & -0.95 & 0.341 \\
\hline-0.23 & -1.36 & 0.91 & -0.39 & 0.695 \\
\hline-3.23 & -4.95 & -1.51 & -3.69 & 0.000 \\
\hline-8.33 & -11.84 & -4.81 & -4.64 & 0.000 \\
\hline-2.20 & -2.70 & -1.71 & -8.81 & 0.000 \\
\hline
\end{tabular}

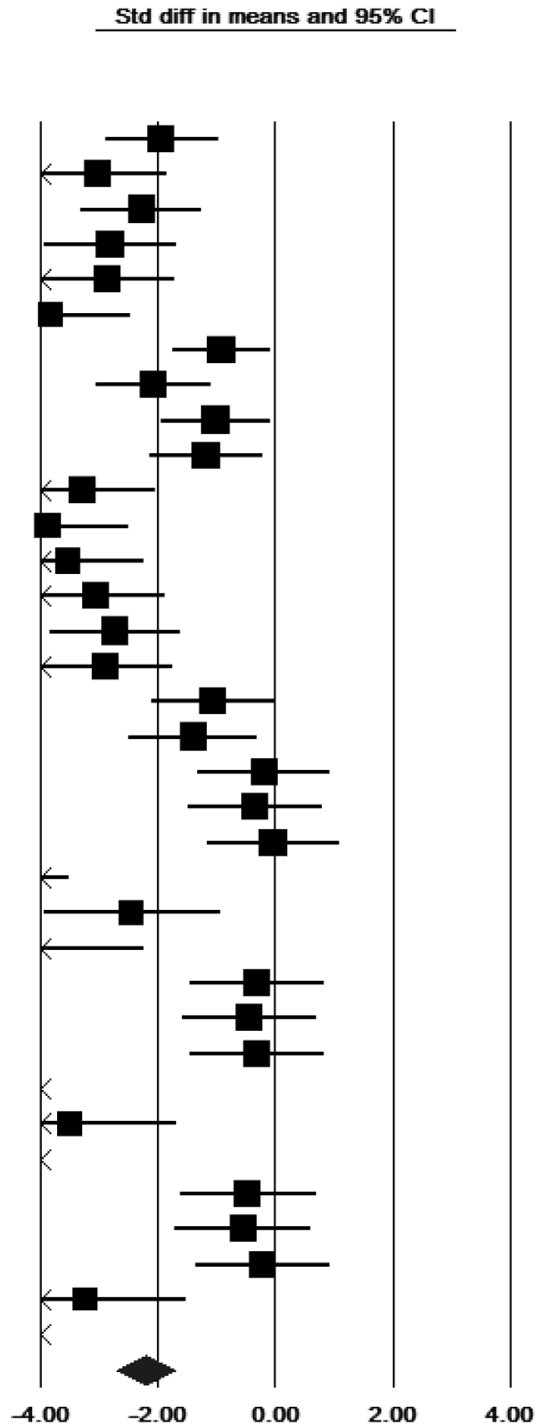

Favors control

Favors DFM

Figure 2. Forest plot for the effect of direct-fed microbial (DFM) supplementation on the log concentrations of Salmonella in the gut of broiler chickens. For each comparison, a black box and its horizontal line represent the point estimate and its 95\% confidence interval (CI) of a standardized mean difference (SMD), respectively. The box or its horizontal line that crosses the middle vertical line (the line of 0.00 ) indicates a non-significant result. The size of the box was proportional to the weight used in meta analysis; the bigger the box, the more weight. The diamond at the bottom of the forest plot represents the overall SMD or the summary effect from all comparisons. The center of the diamond and its lateral tips represent the point estimate and its $95 \% \mathrm{Cl}$ of the overall SMD, respectively.

\section{Assessment of risk of bias}

Overall results from the assessment of risk of bias for the 42 studies for each domain are presented in Figure 3 (results from the assessment for each article are available in Supplementary Table S2). For sequence generation (domain 1), 36 of the 42 articles (86\%) had unclear risks of bias, and six articles (14\%) had high risks of bias. For baseline characteristics (domain 2), 23 of the 42 articles (55\%) had low risks of bias, and 19 articles (45\%) had unclear risks of bias. For allocation concealment (domain 3), 36 of the 42 articles (86\%) had unclear risks of bias. For random housing (domain 4), 30 of the 42 articles (71\%) had unclear risks of bias, and 12 articles (29\%) had low risks of bias. For blinding of the caregivers (domain 5), all 42 articles had unclear risks of bias. For random-outcome assessment (domain 6), 20 of the 42 articles (48\%) had high risks of bias, and 22 articles (52\%) had low risks of bias. For blinding of outcome assessors (domain 7), all 42 articles had low risks of bias. For incomplete outcome data (domain 8), 41 of the 42 articles (98\%) had low risks of bias, and one article (2\%) had an unclear risk of bias. For selective outcome reporting (domain 9) and other sources of bias (domain 10), all 42 articles had unclear risks of bias.

Assessment of publication bias 
Table 2. Subgroup analysis of the effect of DFM supplementation on the log concentrations of Lactobacillus in broiler chickens

\begin{tabular}{|c|c|c|c|c|c|c|c|c|}
\hline \multirow{2}{*}{$\begin{array}{l}\text { Characteristics and } \\
\text { subgroups }\end{array}$} & \multicolumn{4}{|c|}{ Effect size } & \multicolumn{3}{|c|}{ Heterogeneity } & \multirow{2}{*}{$\begin{array}{l}p \text { value for } \\
\text { subgroup } \\
\text { difference }\end{array}$} \\
\hline & $n^{1)}$ & SMD & {$[95 \% \mathrm{Cl}]$} & $p$ value & Q & $p$ value & $I^{2}$ & \\
\hline \multicolumn{9}{|l|}{ Breed } \\
\hline Arbor Acres & 89 & 0.99 & {$[0.71,1.28]$} & $<0.001$ & 370.90 & $<0.001$ & 76 & $<0.001$ \\
\hline Cobb & 18 & 0.91 & {$[0.34,1.48]$} & 0.002 & 89.25 & $<0.001$ & 81 & \\
\hline Cuban EB24 & 6 & 2.13 & {$[0.85,3.42]$} & 0.001 & 39.21 & $<0.001$ & 87 & \\
\hline Lingnan Yellow & 18 & 0.57 & {$[0.22,0.91]$} & 0.001 & 26.11 & 0.073 & 35 & \\
\hline Ross & 46 & 1.21 & {$[0.87,1.54]$} & $<0.001$ & 235.87 & $<0.001$ & 81 & \\
\hline Sasso X44 & 8 & 0.63 & {$[-0.11,1.38]$} & 0.096 & 20.77 & 0.004 & 66 & \\
\hline Tsukuba jidori & 6 & -0.17 & {$[-0.68,0.34]$} & 0.513 & 2.68 & 0.749 & 00 & \\
\hline Unknown & 14 & 1.02 & {$[-0.20,2.24]$} & 0.102 & 70.48 & $<0.001$ & 82 & \\
\hline \multicolumn{9}{|l|}{$\mathrm{Sex}^{22}$} \\
\hline Both & 30 & 1.26 & {$[0.88,1.65]$} & $<0.001$ & 76.17 & $<0.001$ & 62 & 0.077 \\
\hline Male & 101 & 1.07 & {$[0.80,1.33]$} & $<0.001$ & 481.64 & $<0.001$ & 79 & \\
\hline Unknown & 71 & 0.74 & {$[0.44,1.03]$} & $<0.001$ & 317.40 & $<0.001$ & 78 & \\
\hline \multicolumn{9}{|l|}{ DFM product } \\
\hline Commercial & 68 & 0.89 & {$[0.58,1.19]$} & $<0.001$ & 333.85 & $<0.001$ & 80 & 0.570 \\
\hline Non-commercial & 137 & 0.99 & {$[0.78,1.21]$} & $<0.001$ & 568.70 & $<0.001$ & 76 & \\
\hline \multicolumn{9}{|l|}{ Microbial species } \\
\hline Multiple & 58 & 0.56 & {$[0.31,0.81]$} & $<0.001$ & 183.41 & $<0.001$ & 69 & 0.004 \\
\hline Single & 142 & 1.09 & {$[0.87,1.32]$} & $<0.001$ & 636.58 & $<0.001$ & 78 & \\
\hline Unknown & 5 & 2.38 & {$[0.02,4.73]$} & 0.048 & 55.90 & $<0.001$ & 93 & \\
\hline \multicolumn{9}{|l|}{ Sampling organ ${ }^{3)}$} \\
\hline Crop & 12 & 1.75 & {$[0.88,2.63]$} & $<0.001$ & 37.68 & $<0.001$ & 71 & 0.386 \\
\hline Ileum & 38 & 0.72 & {$[0.29,1.15]$} & 0.001 & 191.67 & $<0.001$ & 81 & \\
\hline Caecum & 110 & 0.90 & {$[0.68,1.12]$} & $<0.001$ & 466.25 & $<0.001$ & 77 & \\
\hline Colon & 14 & 1.23 & {$[0.02,2.43]$} & 0.046 & 71.51 & $<0.001$ & 82 & \\
\hline Excreta & 7 & 1.20 & {$[0.11,2.28]$} & 0.030 & 51.95 & $<0.001$ & 88 & \\
\hline Unknown & 21 & 1.09 & {$[0.52,1.62]$} & $<0.001$ & 51.45 & $<0.001$ & 61 & \\
\hline \multicolumn{9}{|l|}{ Application route ${ }^{4)}$} \\
\hline Feed & 191 & 0.96 & {$[0.77,1.14]$} & $<0.001$ & 863.92 & $<0.001$ & 78 & 0.785 \\
\hline Water & 9 & 0.73 & {$[-0.10,1.55]$} & 0.085 & 19.06 & 0.015 & 58 & \\
\hline Gavage & 4 & 1.36 & {$[-0.44,3.16]$} & 0.139 & 17.08 & 0.001 & 82 & \\
\hline \multicolumn{9}{|l|}{ Application frequency } \\
\hline Daily & 201 & 0.95 & {$[0.77,1.013]$} & $<0.001$ & 885.43 & $<0.001$ & 77 & 0.658 \\
\hline Once & 4 & 1.36 & {$[-0.44,3.16]$} & 0.139 & 17.08 & 0.001 & 82 & \\
\hline \multicolumn{9}{|c|}{$\begin{array}{l}\text { Application duration(days of } \\
\text { age) }\end{array}$} \\
\hline$\leq 7$ & 29 & 0.64 & {$[0.31,0.97]$} & $<0.001$ & 51.01 & 0.005 & 45 & $<0.001$ \\
\hline$>7, \leq 14$ & 26 & 0.47 & {$[0.11,0.83]$} & 0.011 & 58.73 & $<0.001$ & 57 & \\
\hline$>14, \leq 21$ & 49 & 1.05 & {$[0.57,1.53]$} & $<0.001$ & 285.76 & $<0.001$ & 83 & \\
\hline$>21, \leq 42$ & 95 & 1.23 & {$[0.98,1.49]$} & $<0.001$ & 477.93 & $<0.001$ & 80 & \\
\hline$>42$ & 6 & -0.17 & {$[-0.68,0.36]$} & 0.513 & 2.68 & 0.749 & 00 & \\
\hline
\end{tabular}

DFM, direct-fed microbial; SMD, standardized mean difference; $\mathrm{Cl}$, confidence interval.

${ }^{1)} n$, number of comparisons.

${ }^{2)}$ Three comparisons of "Female" sex were excluded from the subgroup analysis.

${ }^{3)}$ Three comparisons of "Duodenum" sampling organ were excluded from the subgroup analysis.

4) One comparison of "Feed and water" application route was excluded from the subgroup analysis.

The results of the assessment of publication bias for each category of taxa are presented in Figures 4-6. In category 1 (taxa whose log concentrations did not differ significantly from the controls, $\mathrm{n}=130$ comparisons), publication bias was not apparent in the funnel plot (Figure 4), and Egger's test was not significant $(\mathrm{p}=0.182)$. In category 2 (taxa whose log concentrations increased significantly with DFM supplementation, $\mathrm{n}=279$ comparisons), the funnel plot clearly indicated publication bias (Figure 5), and Egger's test was significant ( $\mathrm{p}<0.001$ ). Duval and Tweedie's trim and fill methods indicated 62 miss- 
Table 3. Subgroup analysis of the effect of DFM supplementation on the log concentrations of coliforms and Escherichia coli in broiler chickens

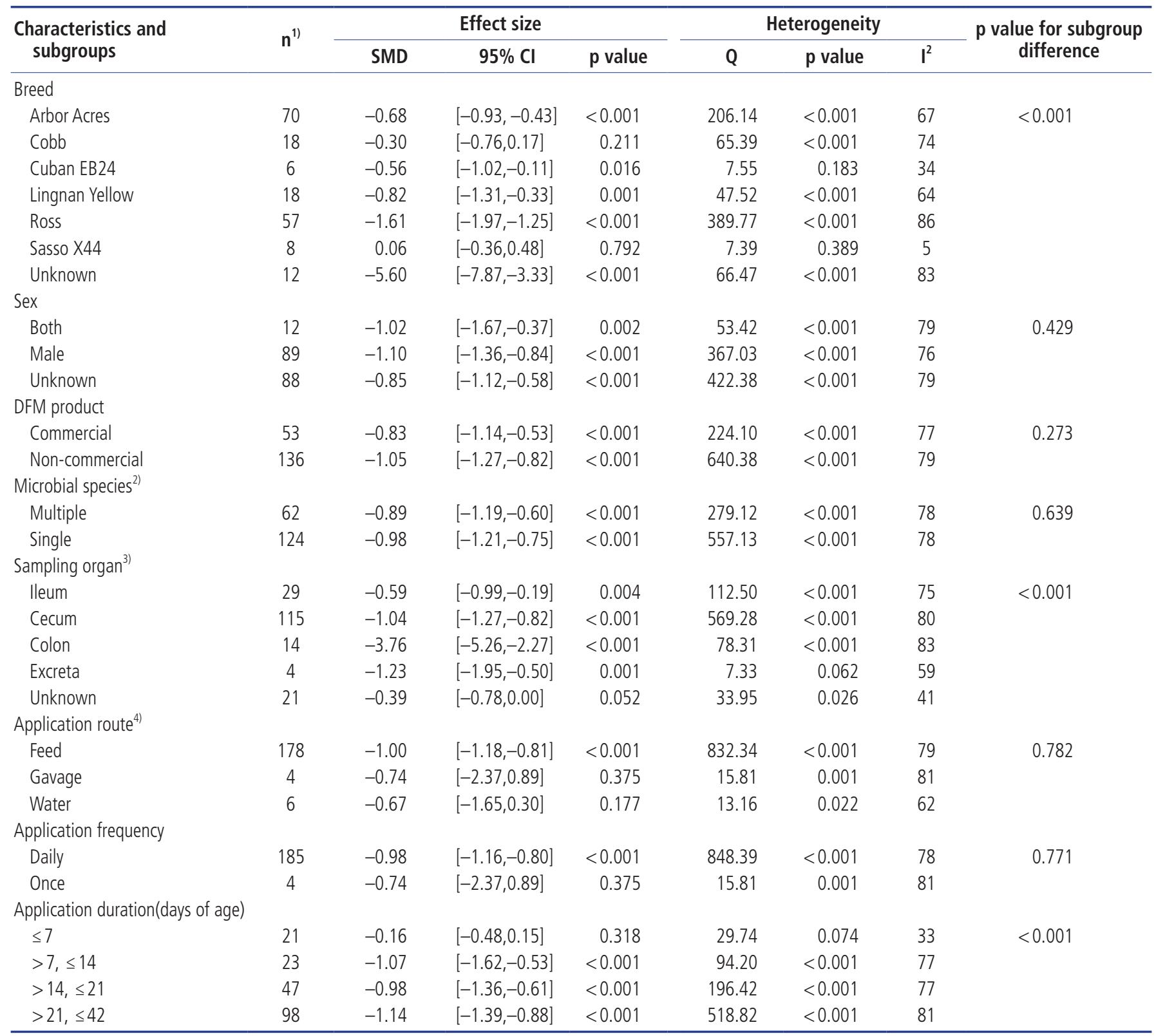

DFM, direct-fed microbial; SMD, standardized mean difference; $\mathrm{Cl}$, confidence interval.

1) $n$, number of comparisons.

${ }^{2)}$ Three comparisons of "Unknown" microbial species were excluded from the subgroup analysis.

3) Three comparisons of "Crop" and three comparisons of "Duodenum" sampling organs were excluded from the subgroup analysis.

4) One comparison of "Feed and water" application route was excluded from the subgroup analysis.

ing comparisons for this category, which adjusted SMD to 0.54 (95\% CI, 0.37 to 0.71 ) from the original SMD of 1.07 (95\% CI, 0.92 to 1.22 ). In category 3 (taxa whose log concentrations decreased significantly with DFM supplementation, $\mathrm{n}=290$ comparisons), the funnel plot clearly indicated publication bias (Figure 6), and Egger's test for publication bias was significant $(\mathrm{p}<0.001)$.

\section{DISCUSSION}

Our meta-analysis suggests that DFM supplementation can confer health benefits to broiler chickens by increasing the concentrations of some beneficial bacteria and decreasing those of some detrimental bacteria. DFM supplementation did not significantly alter the overall log concentrations of culturable gut microbiotas, including total bacterial counts, but it significantly increased the log concentrations of four taxa (Bacillus, Bifidobacterium, C. butyricum, and Lactobacillus) and significantly decreased those of five taxa (C. perfringens, coliforms, E. coli, Enterococcus, and Salmonella). 
Table 4. Sensitivity analysis for comparing random-and fixed-effects models

\begin{tabular}{|c|c|c|c|c|c|c|c|}
\hline \multirow{2}{*}{ Model } & \multirow{2}{*}{$n^{1)}$} & \multicolumn{3}{|c|}{ Effect size } & \multicolumn{3}{|c|}{ Heterogeneity } \\
\hline & & SMD & {$[95 \% \mathrm{Cl}]$} & $p$ value & Q & $p$ value & $\mathrm{I}^{2}$ \\
\hline \multicolumn{8}{|l|}{ Random effect } \\
\hline Category 12) & 130 & -0.12 & {$[-0.35,0.11]$} & 0.298 & 592.62 & $<0.001$ & 78 \\
\hline Category $2^{3)}$ & 279 & 1.07 & {$[0.92,1.22]$} & $<0.001$ & $1,283.64$ & $<0.001$ & 78 \\
\hline (ategory $3^{4)}$ & 290 & -1.26 & {$[-1.42,-1.10]$} & $<0.001$ & $1,526.73$ & $<0.001$ & 81 \\
\hline \multicolumn{8}{|l|}{ Fixed effect } \\
\hline Category 1 & 130 & -0.08 & {$[-0.19,0.02]$} & 0.116 & 592.62 & $<0.001$ & 78 \\
\hline Category 2 & 279 & 0.77 & {$[0.70,0.83]$} & $<0.001$ & $1,283.64$ & $<0.001$ & 78 \\
\hline Category 3 & 290 & -0.89 & {$[-0.95,-0.82]$} & $<0.001$ & $1,526.73$ & $<0.001$ & 81 \\
\hline
\end{tabular}

SMD, standardized mean difference; $\mathrm{Cl}$, confidence interval.

1) $n$, number of comparisons.

${ }^{2)}$ Category 1, bacteria whose log concentrations did not differ significantly from the controls.

${ }^{3)}$ Category 2, bacteria that their log concentrations increased significantly with DFM supplementation.

4) Category 3, bacteria whose log concentrations decreased significantly with DFM supplementation.

In the group whose log concentrations increased significantly, Bifidobacterium and Lactobacillus, genera predominantly found in the gastrointestinal tracts of animals, including chickens, are beneficial to the host and generally regarded as safe [15]. Increasing their concentrations would therefore confer health benefits to the host, which has led to extensive investigation of their probiotic potentials in a variety of animal species $[16,17]$. Twenty-three of the 42 studies used Lactobacillus in DFM supplementation, indicating its popularity for application in broiler chickens. Lactobacillus, a Gram-positive, facultative anaerobic or microaerophilic, rod-shaped, nonspore-forming bacterium belonging to the phylum Firmicutes, has several beneficial effects on the host, e.g. producing digestive enzymes, helping to breakdown bile salts, helping the synthesis of vitamins $B$ and $\mathrm{K}$, and enhancing innate and acquired immunity [18]. Our results of increased concentrations of Lactobacillus were consistent with those in other studies, such as in pigs [19], dogs [20], and mice [21]. Whether the increased concentrations of Lactobacillus were due to DFM supplementation or to indigenous bacteria, however, remains unclear because of study limitations identifying bacterial origins.

Only 5 of the 42 studies used Bifidobacterium in DFM supplementation, indicating its lower popularity for application in broiler chickens. The lower popularity may be due to the

1. Sequence generation (Selection bias)

2. Baseline characteristics (Selection bias)

3. Allocation concealment (Selection bias)

4. Random housing (Performance bias)

5. Blinding (Performance bias)

6. Random outcome assessment (Detection bias)

7. Blinding (Detection bias)

8. Incomplete outcome data (Attrition bias)

9. Selective outcome reporting (Reporting bias)

10. Other sources of bias

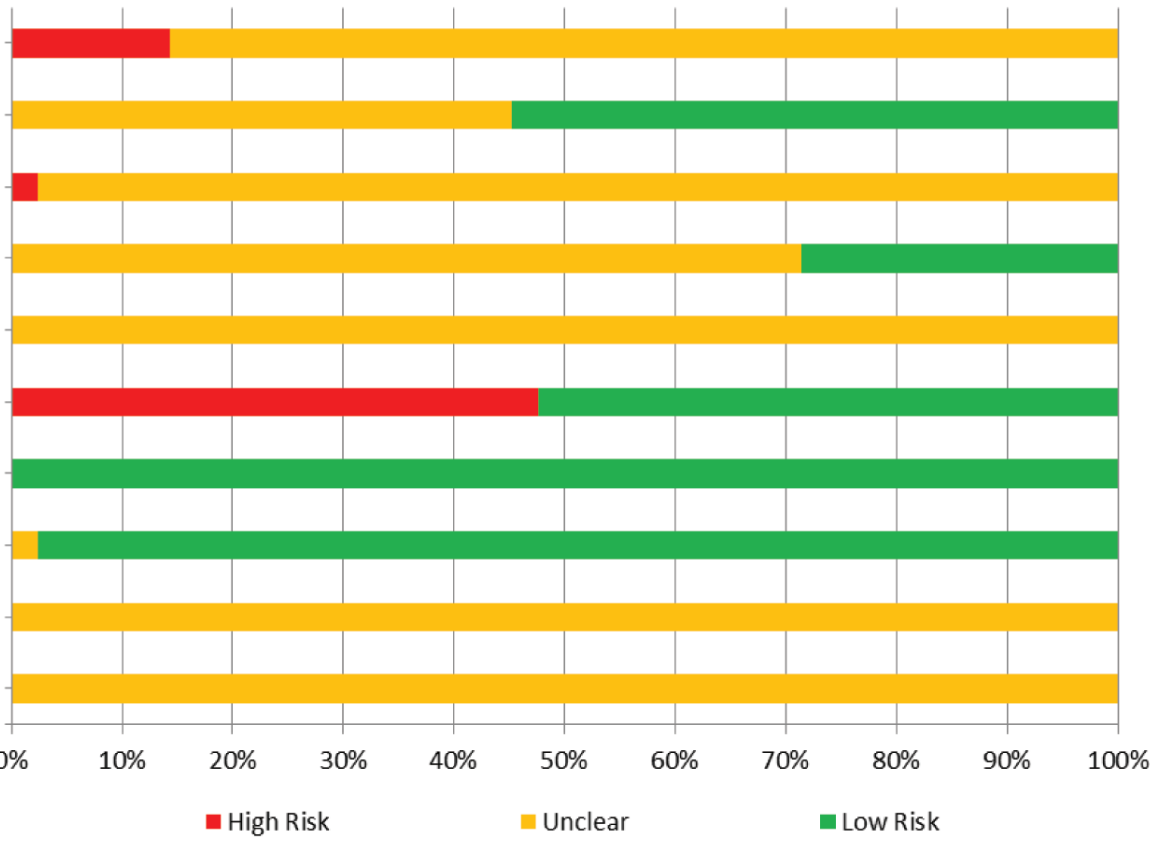

Figure 3. Risk of bias presented as the percentage of the 42 studies. All or almost all articles had unclear risk of bias for domains 1, 3, 5, 9, and 10, indicating insufficient information from the articles to determine the risk status for those domains. Blinding for detection bias (domain 7) was considered low risk for all articles because the log concentration of the bacterial count was not a subjective outcome. 


\section{Funnel Plot of Precision by Std diff in means}

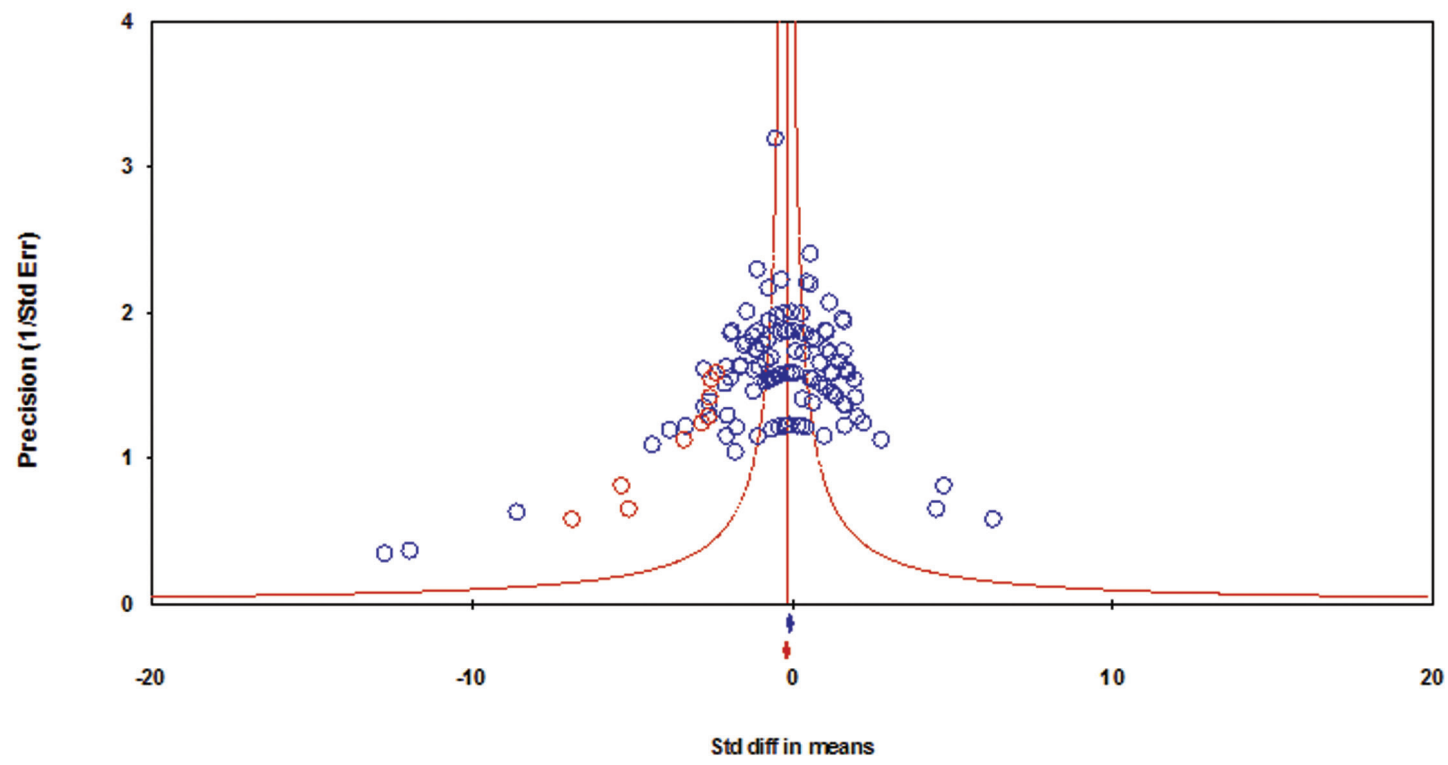

Figure 4. Funnel plot for bacteria in category 1 (taxa whose log concentrations did not differ significantly from the controls). Y-axis represents the study precision (the inverse of standard error) and $x$-axis shows the standardized mean difference (SMD). A blue circle represents each comparison $(n=130)$ of the included studies. Distribution of the blue circles was approximate symmetry around a red vertical line (the line corresponding to the overall SMD), indicating that publication bias was not apparent. Only nine missing comparisons (red circles) were imputed as suggested by trim and fill analysis. A blue and a red marker at the bottom of the funnel plot represent the overall SMD before and after missing comparisons were imputed, respectively.

lower abundance of Bifidobacterium than Lactobacillus in the gastrointestinal tract of chickens [2]. Bifidobacterium, a Grampositive, non-motile, often branched anaerobic bacterium belonging to the phylum Actinobacteria, however, is very important in humans because it is found predominantly in infants [22]. A significant change in bifidobacterial number or composition is associated with several gastrointestinal disorders in humans, such as irritable bowel syndrome and inflamma-

\section{Funnel Plot of Precision by Std diff in means}

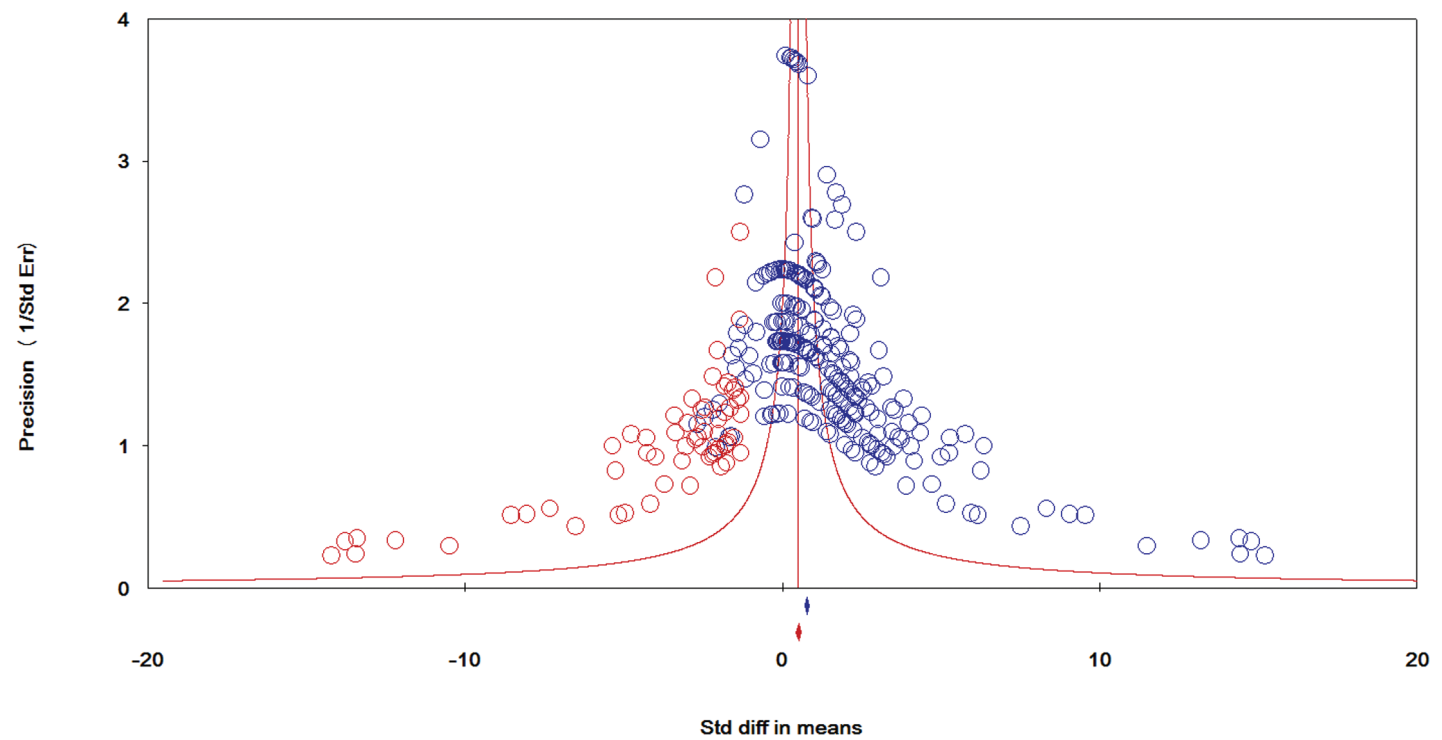

Figure 5. Funnel plot for bacteria in category 2 (taxa whose log concentrations increased significantly with DFM supplementation). Y-axis represents the study precision (the inverse of standard error) and $x$-axis shows the standardized mean difference (SMD). A blue circle represents each comparison $(n=279)$ of the included studies. Distribution of the blue circles was clearly asymmetric around a red vertical line (the line corresponding to the overall SMD), indicating that publication bias was apparent. As many as 62 missing comparisons (red circles) were imputed on the left hand side of the plot as suggested by trim and fill analysis. A blue and a red marker at the bottom of the funnel plot represent the overall SMD before and after missing comparisons were imputed, respectively. 


\section{Funnel Plot of Precision by Std diff in means}

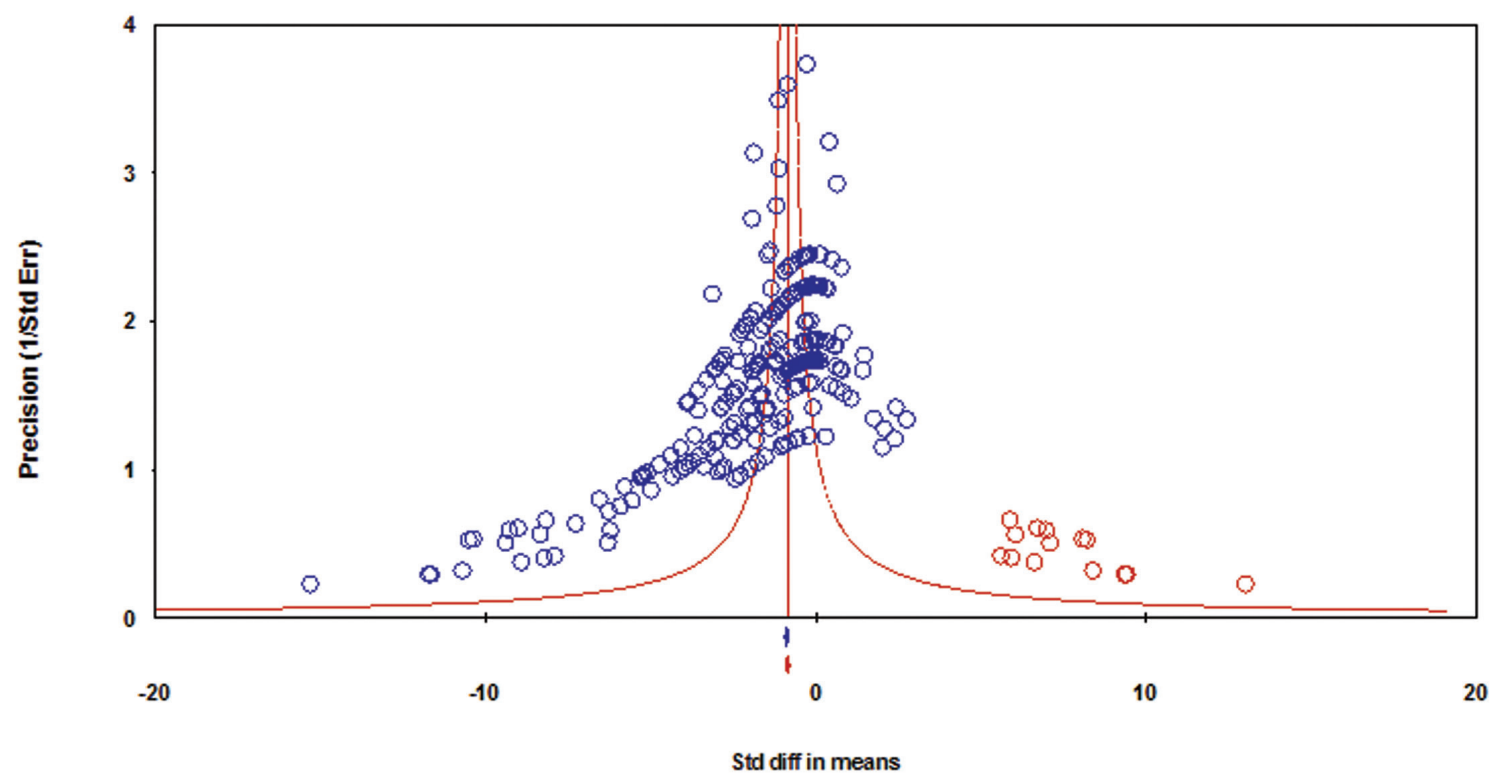

Figure 6. Funnel plot for bacteria in category 3 (taxa whose log concentrations decreased significantly with DFM supplementation). Y-axis represents the study precision (the inverse of standard error) and $x$-axis shows the standardized mean difference (SMD). A blue circle represents each comparison $(n=290)$ of the included studies. Distribution of the blue circles was clearly asymmetric around a red vertical line (the line corresponding to the overall SMD), indicating that publication bias was apparent. Fourteen missing comparisons (red circles) were imputed on the right hand side of the plot as suggested by trim and fill analysis. A blue and a red marker at the bottom of the funnel plot represent the overall SMD before and after missing comparisons were imputed, respectively.

tory bowel disease [23]; many Bifidobacterium species and strains have therefore been identified and used as probiotics in humans [24].

Sixteen of the 42 studies used Bacillus in DFM supplementation, indicating moderate application in broiler chickens. Bacillus is a Gram-positive, rod-shaped, endospore-forming, aerobic or facultatively anaerobic bacterium belonging to the phylum Firmicutes. Bacillus is of great interest as a probiotic candidate in broiler chickens because it can produce durable spores that can germinate in extreme environments such as gastrointestinal tracts and during food processing. Bacillus can also produce various beneficial substances such as antimicrobial compounds, enzymes, and vitamins [25]. Only two species of Bacillus were used in the 15 studies; 13 used Bacillus subtilis (B. subtilis) and two used Bacillus amyloliquefaciens, indicating the importance of $B$. subtilis as a DFM product in broiler chickens. Some Bacillus species, though, are pathogenic, such as Bacillus anthracis (the causative agent of anthrax) and Bacillus cereus (a causative agent of food poisoning). The pros and cons of the Bacillus genus have raised safety concerns for its use in probiotics, so individual Bacillus strains or species must be evaluated for safety for each application [25]. Information for Bacillus was available in our study at the genus level, so the health benefits from increasing the log concentrations of Bacillus remained unclear.

Several mechanisms have been proposed for the alteration or modulation of gut microbiota by DFMs or probiotics. DFMs can secrete antimicrobial agents or other metabolic agents that suppress the growth of other microbes or compete for binding sites on the gut mucosa [26], balancing beneficial and detrimental microbes in the gut. This balance or homeostasis is very important for host health. Unbalanced gut microbiotas (dysbiosis or dysbacteriosis) [27] are associated with several gastrointestinal diseases, such as necrotic enteritis in chickens [28]. Many factors other than DFM can determine or alter the gut microbiota in chickens, e.g. dietary components, antibiotic growth promoters, prebiotics [29], feeding patterns [30], and litter [31]. Detailed contents of these factors are beyond the scope of this discussion. A review by Stanley et al [2] provide excellent information.

DFM supplementation has been strongly associated with increasing beneficial bacteria and decreasing detrimental bacteria in the gut of broiler chickens, but our results indicated high heterogeneity among studies, even after subgroup analysis, i.e. the effects of DFM supplementation varied from one study to another.

The results from the subgroup analysis indicated that the effectiveness of DFM supplementation may differ among the subgroups of some characteristics. The subgroup differences were found in three characteristics (broiler breed, microbial species, and application duration) for the log concentrations of Lactobacillus (Table 2) and three characteristics (broiler breed, sampling organ, and application duration) for those of coliforms and E. coli (Table 3). For the log concentrations of 
Lactobacillus, Cuban EB24 and Ross were associated with greater effect size. However, the estimated effect size for Cuban EB24 was not precise because a CI of the estimate was substantially wide with the very small number of studies $(n=6)$. For the log concentrations of coliforms and E. coli, Ross and unknown breeds were associated with greater effect size. We found that 14 studies reported unknown breeds for the subgroup comparisons; as a result, this caused a difficulty in interpretation due to the limited information about the breeds. The discrepancy or heterogeneity on the log concentrations of Lactobacillus and a combination of coliforms and E. coli among breeds of chickens can be explained that the genetic background (chicken type and breed) has been considered as a factor influencing the gut microbiota composition [32]. In turn, the gut microbiota from each breed or even individual animals may interact differently with DFM supplementation. This interaction along with other influencing factors may result in the different response from each breed or individual animals. Surprisingly, we found that increase in the log concentrations of Lactobacillus was greater in the application of single DFM species than that of multiple DFM species. These results were opposite to our expectation because many studies showed that an application of multiple DFM or multiple probiotic species would get better results $[33,34]$. These unexpected results could be explained that most trials in broiler chickens used Lactobacillus-based DFM with known and higher concentrations than multiple species-based DFM. The log concentrations of Lactobacillus detected in the samples came from a combination of the indigenous bacteria and the bacteria of DFM supplementation. Application duration between 21 and 42 days of age was associated with the greatest increase in the log concentrations of Lactobacillus and with the greatest decrease in those of coliforms and E. coli. These findings indicated that DFM supplementation would help to reduce a risk of detrimental bacterial contaminations in commercial broiler products. The age of chickens and the time point at which DFM supplementation is administered are well-known factors for its effectiveness [35]. Additional subgroup analysis was performed on DFM supplementation at a genus level (results are present in Supplementary Table S3-S4 of the supplementary data). A significant subgroup difference was observed for both the log concentrations of Lactobacillus and those of coliforms and E. coli. This indicated that genus, species, or even strains of DFM may be associated with the effectiveness. In addition, many other factors (including environment factors) may affect the gut microbiota in broiler chickens. These factors included housing, feed, litter, hygiene, climate and geographical location [36]. It is possible that these factors may also influence the effectiveness of DFM supplementation. The residual heterogeneity within each subgroup for each character after the subgroup analysis, however, was still high (Tables 3,4), indicating that other (unknown) factors were responsible for the heterogeneity. Heterogeneous effects of DFMs or probiotics have been identified in several metaanalyses, e.g. the effect of probiotic supplementation on growth performance in broiler chickens [37]. This variability may account for the low acceptance of DFM or probiotic supplementation as a routine practice in the poultry industry, compared with other interventions such as antibiotics.

Our study has several limitations that should be taken into account when interpreting the results. First, the measured outcome is specific to the log concentration of the culturable gut microbiota. The majority of the gut microbiota cannot be cultured, so the results of this study cannot represent the entire microbiota but just some species of interest of culturable microbiota that have been reported. The use of techniques not based on culturability, especially next-generation sequencing, will allows us to better understand the abundance and diversity of the gut microbiota. This information, however, is currently too limited in trials of DFM supplementation in broiler chickens to synthesize the data. Second, the heterogeneity of outcomes is high even after accounting for subgroup analysis. Results are thus quite variable among studies, and characteristics other than our pre-specified characteristics in the subgroup analysis were responsible for the remaining heterogeneity. Third, most studies did not have clear risks of bias for most of the domains assessed, indicating that insufficient information was available for judging the bias. Our conclusions may change if new information indicates clear risks of bias. Fourth, publication bias was clear in the outcome for categories 2 (taxa whose log concentrations increased significantly with DFM supplementation) and 3 (taxa whose log concentrations decreased significantly with DFM supplementation) but not 1 (taxa whose log concentrations did not differ significantly from the controls). Publication bias arises from many sources and can distort the effect size of the outcome under study [38].

In conclusion, this systematic review and meta-analysis found that DFM supplementation modulated the gut microbiotas of broiler chickens by increasing the log concentrations of beneficial bacteria (Bacillus, Bifidobacterium, C. butyricum, and Lactobacillus) and decreasing those of detrimental bacteria (C. perfringens, coliforms, E. coli, Enterococcus, and Salmonella), suggesting health benefits of DFM supplementation for broiler chickens. This conclusion, however, was based on highly heterogeneous results among the studies, unclear risks of bias for reporting quality assessment, and publication bias of the available data in reporting bacterial taxa with statistically significant tests.

\section{CONFLICT OF INTEREST}

We certify that there is no conflict of interest with any financial organization regarding the material discussed in the manuscript. 


\section{ACKNOWLEDGMENTS}

The authors greatly appreciate the support from the program "Royal Scholarship under Her Royal Highness Princess Maha Chakri Sirindhorn Education Project", and from the Faculty of Veterinary Medicine, Khon Kaen University.

\section{REFERENCES}

1. Buntyn JO, Schmidt TB, Nisbet DJ, Callaway TR. The role of direct-fed microbials in conventional livestock production. Annu Rev Anim Biosci 2016;4:335-55.

2. Stanley D, Hughes RJ, Moore RJ. Microbiota of the chicken gastrointestinal tract: influence on health, productivity and disease. Appl Microbiol Biotechnol 2014;98:4301-10.

3. Hermans D, Pasmans F, Messens W, et al. Poultry as a host for the zoonotic pathogen Campylobacter jejuni. Vector Borne Zoonotic Dis 2012;12:89-98.

4. Lei X, Piao X, Ru Y, et al. Effect of Bacillus amyloliquefaciensbased direct-fed microbial on performance, nutrient utilization, intestinal morphology and cecal microflora in broiler chickens. Asian-Australas J Anim Sci 2015;28:239-46.

5. Choi KY, Lee TK, Sul WJ. Metagenomic Analysis of chicken gut microbiota for improving metabolism and health of chickens - a review. Asian-Australas J Anim Sci 2015;28:1217-25.

6. Oakley BB, Lillehoj HS, Kogut MH, et al. The chicken gastrointestinal microbiome. FEMS Microbiol Lett 2014;360:100-12.

7. de Vries RBM, Hooijmans CR, Langendam MW, et al. A protocol format for the preparation, registration and publication of systematic reviews of animal intervention studies. Evid Based Preclin Med 2015;2:1-9.

8. Kerr AK, Farrar AM, Waddell LA, et al. A systematic reviewmeta-analysis and meta-regression on the effect of selected competitive exclusion products on Salmonella spp. prevalence and concentration in broiler chickens. Prev Vet Med 2013;111: $112-25$.

9. Blajman JE, Frizzo LS, Zbrun MV, et al. Probiotics and broiler growth performance: a meta-analysis of randomised controlled trials. Br Poult Sci 2014;55:483-94.

10. Hooijmans CR, Rovers MM, de Vries RB, et al. SYRCLE's risk of bias tool for animal studies. BMC Med Res Methodol 2014; 14:43.

11. Higgins JP, Thompson SG, Deeks JJ, Altman DG. Measuring inconsistency in meta-analyses. BMJ 2003;327:557-60.

12. Hooijmans CR, de Vries RB, Rovers MM, Gooszen HG, RitskesHoitinga M. The effects of probiotic supplementation on experimental acute pancreatitis: a systematic review and metaanalysis. PLoS ONE 2012;7:e48811.

13. Egger M, Smith GD, Schneider M, Minder C. Bias in metaanalysis detected by a simple, graphical test. BMJ 1997;315: 629-34.

14.Duval S, Tweedie R. Trim and fill: A simple funnel-plot-based method of testing and adjusting for publication bias in metaanalysis. Biometrics 2000;56:455-63.

15. Butel MJ. Probiotics, gut microbiota and health. Med Mal Infect 2014;44:1-8.

16. Surachon P, Sukon P, Chaveerach P, Waewdee P, Soikum C. Screening of lactic acid bacteria isolated from chicken ceca for in vitro growth inhibition of Salmonella enteritica serovar enteritidis. J Anim Vet Adv 2011;10:939-44.

17.Valdes-Varela L, Alonso-Guervos M, Garcia-Suarez O, Gueimonde M, Ruas-Madiedo P. Screening of bifidobacteria and lactobacilli able to antagonize the cytotoxic effect of Clostridium difficile upon intestinal epithelial HT29 monolayer. Front Microbiol 2016;7:577.

18. Alok A, Singh ID, Singh S, et al. Probiotics: A new era of biotherapy. Adv Biomed Res 2017;6:31.

19. Lan R, Koo J, Kim I. Effects of Lactobacillus acidophilus supplementation on growth performance, nutrient digestibility, fecal microbial and noxious gas emission in weaning pigs. J Sci Food Agric 2017;97:1310-5.

20. Strompfova V, Kubasova I, Laukova A. Health benefits observed after probiotic Lactobacillus fermentum CCM 7421 application in dogs. Appl Microbiol Biotechnol 2017;101:6309-19.

21.Jeong D, Kim DH, Kang IB, et al. Modulation of gut microbiota and increase in fecal water content in mice induced by administration of Lactobacillus kefiranofaciens DN1. Food Funct 2017;8:680-6.

22. Turroni F, Peano C, Pass DA, et al. Diversity of bifidobacteria within the infant gut microbiota. PLoS ONE 2012;7:e36957.

23. Tojo R, Suarez A, Clemente MG, et al. Intestinal microbiota in health and disease: role of bifidobacteria in gut homeostasis. World J Gastroenterol 2014;20:15163-76.

24.Sarkar A, Mandal S. Bifidobacteria-Insight into clinical outcomes and mechanisms of its probiotic action. Microbiol Res 2016;192:159-71.

25. Elshaghabee FMF, Rokana N, Gulhane RD, Sharma C, Panwar H. Bacillusas potential probiotics: status, concerns, and future perspectives. Front Microbiol 2017;8:1490.

26. Hemarajata P, Versalovic J. Effects of probiotics on gut microbiota: mechanisms of intestinal immunomodulation and neuromodulation. Therap Adv Gastroenterol 2013;6:39-51.

27.Ducatelle R, Eeckhaut V, Haesebrouck F, Van Immerseel F. A review on prebiotics and probiotics for the control of dysbiosis: present status and future perspectives. Animal 2015;9:43-8.

28. Antonissen G, Eeckhaut V, Van Driessche K, et al. Microbial shifts associated with necrotic enteritis. Avian Pathol 2016;45: 308-12.

29. Pan D, Yu Z. Intestinal microbiome of poultry and its interaction with host and diet. Gut Microbes 2014;5:108-19.

30.Cui Y, Wang Q, Liu S, et al. Age-related variations in intestinal microflora of free-range and caged hens. Front Microbiol 2017; 8:1310.

31.Wang L, Lilburn M, Yu Z. Intestinal microbiota of broiler 
chickens as affected by litter management regimens. Front Microbiol 2016;7:593.

32. Kers JG, Velkers FC, Fischer EAJ, et al. Host and environmental factors affecting the intestinal microbiota in chickens. Front Microbiol 2018;9:235.

33. Chang H-Y, Chen J-H, Chang J-H, et al. Multiple strains probiotics appear to be the most effective probiotics in the prevention of necrotizing enterocolitis and mortality: An updated meta-analysis. PLoS ONE 2017;12:e0171579.

34. Chapman CMC, Gibson GR, Rowland I. Health benefits of probiotics: are mixtures more effective than single strains? Eur J Nutr 2011;50:1-17.
35.Nakphaichit M, Thanomwongwattana S, Phraephaisarn C, et al. The effect of including Lactobacillus reuteri KUB-AC5 during post-hatch feeding on the growth and ileum microbiota of broiler chickens. Poult Sci 2011;90:2753-65.

36. Clavijo V, Flórez MJV. The gastrointestinal microbiome and its association with the control of pathogens in broiler chicken production: a review. Poult Sci 2018;97:1006-21.

37. Blajman JE, Frizzo LS, Zbrun MV, et al. Probiotics and broiler growth performance: a meta-analysis of randomised controlled trials. Br Poult Sci 2014;55:483-94.

38. Thornton A, Lee P. Publication bias in meta-analysis: its causes and consequences. J Clin Epidemiol 2000;53:207-16. 